\title{
Resolving isospectral 'drums' by counting nodal domains
}

\author{
Sven Gnutzmann ${ }^{1,3}$, Uzy Smilansky ${ }^{2,4}$ and Niels Sondergaard ${ }^{2,5}$ \\ ${ }^{1}$ Institute for Theoretical Physics, Freie Universität Berlin, 14195 Berlin, Germany \\ 2 Department of Physics of Complex Systems, The Weizmann Institute of Science, \\ Rehovot 76100, Israel
}

Received 24 April 2005, in final form 26 August 2005

Published 28 September 2005

Online at stacks.iop.org/JPhysA/38/8921

\begin{abstract}
Several types of systems have been put forward during the past few decades to show that there exist isospectral systems which are metrically different. One important class consists of Laplace-Beltrami operators for pairs of flat tori in $\mathbb{R}^{n}$ with $n \geqslant 4$. We propose that the spectral ambiguity can be resolved by comparing the nodal sequences (the numbers of nodal domains of eigenfunctions, arranged by increasing eigenvalues). In the case of isospectral flat tori in four dimensions-where a four-parameter family of isospectral pairs is known - we provide heuristic arguments supported by numerical simulations to support the conjecture that the isospectrality is resolved by the nodal count. Thus one can count the shape of a drum (if it is designed as a flat torus in four dimensions).
\end{abstract}

PACS numbers: $02.40 . \mathrm{Vh}, 05.45 . \mathrm{Mt}$

Mathematics Subject Classification: 58J50, 58J53

\section{Introduction}

Since Kac posed his famous question: 'can one hear the shape of a drum?' [1], the subject of isospectrality appears in many contexts in the physical and the mathematical literature. This question can be cast in a more general way by considering a Riemannian manifold (with or without boundaries) and the corresponding Laplace-Beltrami operator. (Boundary conditions which maintain the self-adjoint nature of the operator are assumed when necessary.) Kac's question is paraphrased to ask "can one deduce the metric of the surface (or the geometry of the

3 Presently on leave at the Department of Physics of Complex Systems, The Weizmann Institute of Science, Rehovot 76100, Israel.

4 Presently on sabbatical leave at the School of Mathematics, Bristol University, Bristol BS81TW, UK.

5 Present address: Division of Mathematical Physics, Lund Institute of Technology, Lund University, Box 118, SE-221 00 Lund, Sweden. 
boundary) from the spectrum?'. To the present day, the answer to this question is not known in sufficient detail. An affirmative answer is known to hold for several classes of surfaces and domains [2-4] (see also a recent review by Zelditch [5]). However, this is not always true. One of the first examples to the negative is due to Milnor who proposed in 1964 two flat tori in $\mathbb{R}^{16}$, which he proved to be isospectral but not isometric [6]. Since then, many other pairs of isospectral yet not isometric systems were found. Fisher considered a discrete version of the Laplacian, and gave a few examples of distinct graphs which share the same spectrum [7]. A general method for constructing isospectral, non-isometric manifolds has been designed by Sunada [10]. Sunada's technique applies also to discrete graphs [11], and the late Brooks [11] gave a few examples of families of non-Sunada discrete graphs. Sunada's method was also used by Gordon et al [8] and Chapman [9] to construct isospectral domains in $\mathbb{R}^{2}$. Other pairs of isospectral domains in $\mathbb{R}^{2}$ were proposed in [13] and were discussed further in [14]. Sunada-like quantum graphs were presented in [4].

Milnor's original work on isospectral flat tori in $\mathbb{R}^{16}$ induced several investigators to find other examples in spaces of lower dimensions. Kneser [15] constructed an example in $n=12$ dimension, and proved that there exist no such pair in two dimensions. Wolpert [16] showed that all sets of mutually isospectral but non-isometric flat tori are finite at any dimension. The first examples in $n=4$ dimension were found by Schiemann [12], and later by Earnest and Nipp [17]. These results were generalized by Conway and Sloane [18], who constructed sets of isospectral pairs of flat tori in $n=4,5,6$, and these sets depend continuously on several parameters.

The existence of such a large variety of isospectral pairs suggests naturally the question: what is the additional information necessary to resolve the isospectrality? We would like to propose that this information is stored in the sequences of nodal counts, defined as follows. Consider only real eigenfunctions of the Laplace-Beltrami operator and assign to each eigenfunction the number of its connected domains where the eigenfunction does not change its sign (such a domain is a nodal domain). The nodal sequence is obtained by arranging the number of nodal domains by the order of increasing eigenvalues. Sturm's oscillation theorem in one dimension and Courant's generalization to higher dimensions express the intimate relation between the nodal sequence and the corresponding spectrum. However, the information stored in the nodal sequence and in the spectrum is not the same, and here we would like to propose that the additional knowledge obtained from the nodal sequence can resolve isospectrality. We address, in particular, isospectral flat tori in four dimensions of the type introduced by Conway and Sloane [18]. The simplicity of the geometry, together with the rich variety of pairs, makes this class of systems very convenient, especially here, when the approach is explored for the first time.

The choice of the nodal sequence to resolve the isospectral ambiguity can be questioned on the grounds that the wave functions depend on the representation where the operator is discussed, in contrast with the spectrum which is independent of the representation. However, looking beyond the spectral information, the nodal sequence seems to be the least problematic choice: it is invariant under all invertible coordinate transformations since nodal lines transform to nodal lines, whose shape might be changed but the number of the domains they enclose remains the same. Moreover, the nodal count has a strong affinity to the spectral sequence expressed by Courant's theorem mentioned above.

Our approach to the study of the nodal sequence is analogous to the one used in spectral statistics. We do not study the detailed structure of the sequence, but rather attempt to find its 'smooth' properties, obtained by averaging over large spectral intervals. This way one loses many details, but gains a description which depends in a simple way on the underlying geometry of the domain under consideration. This approach was used successfully in the past 
[22], and its application in the present context is justified by the results to be reported in the rest of the paper.

This paper is organized as follows. In section (2) we shall summarize some of the properties of flat tori, their spectra and eigenfunctions. In these introductory sections we shall treat the general set of flat tori in $n$ dimensions. However, the concrete examples which we shall treat are in $n=4$, and so are the numerical illustrations. The fact that the spectra are highly degenerate requires a special choice of the basis set of wave functions for which the nodal domains are to be counted. The quantity which signals the difference between the isospectral tori is defined in subsection (3.1). The arguments which lead us to suggest that this quantity resolves isospectrality are explained for the families of flat tori in four dimensions [18] and are presented in subsection (3.2). A summary and some concluding remarks will be given in the last section.

\section{Flat tori}

Consider a set of $n$ linearly independent vectors $\left(\mathbf{g}^{(1)}, \ldots, \mathbf{g}^{(n)}\right) \in \mathbb{R}^{n}$ which span $\mathbb{R}^{n}$. These vectors constitute a unit cell which, upon integer translations, form a lattice. A flat torus consists of the unit cell, in which opposite faces are identified. To be precise, a flat torus is a Riemannian manifold which is a quotient of $\mathbb{R}^{n}$ by a lattice of maximum rank: $T=\mathbb{R}^{n} / A \mathbb{Z}^{n}$, where $A=\left(\mathbf{g}^{(1)}, \ldots, \mathbf{g}^{(n)}\right)$, and the lattice $A \mathbb{Z}^{n}$ is spanned by the $\mathbf{g}^{(r)}$. The reciprocal lattice will be denoted by $\hat{\mathbf{g}}^{(r)}$, and $\left(\hat{\mathbf{g}}^{(s)} \cdot \mathbf{g}^{(r)}\right)=\delta_{r, s}$. The Gram matrices for the lattice will be denoted by $G=A^{\top} A$, and its reciprocal will be denoted for brevity by $Q=G^{-1}=\left(A^{-1}\right)\left(A^{-1}\right)^{\top}$. In the present work we shall deal with dimensions $n \geqslant 4$.

\subsection{Spectra}

We are interested in the spectrum of the Laplace-Beltrami operator $\Delta=-\sum_{i=1}^{n} \frac{\partial^{2}}{\partial x_{i}^{2}}$ with eigenfunctions which are uniquely defined on $T$. They can be explicitly written down as follows.

$$
\Psi_{\mathbf{q}}(\mathbf{x})=\exp \left(2 \pi \mathrm{i} \sum_{r=1}^{n} q_{r}\left(\hat{\mathbf{g}}^{(r)} \cdot \mathbf{x}\right)\right),
$$

where $\mathbf{q}=\left(q_{1}, \ldots, q_{n}\right) \in \mathbb{Z}^{n}, \mathbf{x} \in T$. The corresponding eigenvalues are

$$
E_{\mathbf{q}}=(2 \pi)^{2}(\mathbf{q} \cdot Q \mathbf{q})
$$

The spectrum of a flat torus may be degenerate, and we denote the degeneracy by

$$
g_{Q}(E)=\sharp\left\{\mathbf{q} \in \mathbb{Z}^{n}: E=E_{\mathbf{q}}\right\} .
$$

If the matrix elements of $Q$ are rational, it is convenient to express the eigenvalues in units of $(2 \pi)^{2} / l(Q)$, where $l(Q)$ is the least common denominator of the elements of $Q$. In these units the energy values are integers, and $g_{Q}(E)$ equals the number of times that $E$ can be represented as an integer quadratic form. The integer vectors which satisfy (2) will be called representing vectors in what follows. Their tips are points on the $n$-dimensional ellipsoid (2), and their distribution on the ellipsoid will be discussed below. The study of the spectrum (i.e. those integers that can be represented by a given integer quadratic form) and the degeneracies (the number of representations) is a subject which was studied at length in number theory. Here, we shall give a brief summary of the results which are essential for the present work. The interested reader is referred to $[19,20]$ for further references and details. 
(a) For the integer $Q$, the eigenvalues $E$ are integers, however, not every integer is necessarily in the spectrum [20]. The gaps between successive integers in the spectrum depend delicately on the properties of $Q$. The fluctuations in the spectral density also have a number theoretical origin, and are very hard to follow in detail. It is preferable, therefore, to try to introduce the concept of spectral averages: given a smooth function $F(E), E \in \mathbb{R}$, the spectral average $\langle F(E)\rangle$ is obtained by averaging over the eigenvalues $E_{i}$ in an interval of width $\Delta E$ centered at $E$, with $1 \ll \Delta E \ll E$.

$$
\langle F(E)\rangle=\frac{1}{M} \sum_{i:\left|E_{i}-E\right|<\Delta E / 2} F\left(E_{i}\right),
$$

$M$ stands for the number of different eigenvalues (counted without multiplicity) in the interval $\Delta E$.

(b) The mean degeneracy $\left\langle g_{Q}(E)\right\rangle$ for the integer $Q$ increases as $E^{\frac{n}{2}-1}$. This estimate can be derived by a simple heuristic argument. The number of integer grid points in a shell of width $\Delta E$ is proportional to $E^{\frac{n-1}{2}} \Delta E / E^{\frac{1}{2}}$. If $Q$ is an integer, $E$ must be an integer, and hence, the number of values it can take in the interval of interest is $\Delta E$. Thus,

$$
\left\langle g_{\text {rat }}(E)\right\rangle \sim \frac{\text { Number of grid points }}{\text { Number of possible values }} \propto E^{\frac{n}{2}-1} \text {. }
$$

(c) For irrational matrices of the form $Q=Q_{0}+\alpha Q_{1}$ where $Q_{0}, Q_{1}$ are both integer, and $\alpha$ is irrational, the mean degeneracy increases more slowly with $E$, namely, $g(E) \propto E^{\frac{n}{2}-2}$. Here, the degeneracy class consists of the grid points which satisfy both $E_{0}=\mathbf{q} \cdot Q_{0} \mathbf{q}$ and $E_{1}=\mathbf{q} \cdot Q_{1} \mathbf{q}$ with $E=E_{0}+\alpha E_{1}$, and both $E_{0}$ and $E_{1}$ are integers. Their number is proportional to the volume of the intersection of the corresponding ellipsoid shells $E^{\frac{n-2}{2}} \Delta E_{0} \Delta E_{1} /\left(E_{0} E_{1}\right)^{\frac{1}{2}}$. The number of spectral values is now the number of integer points in the square of size $\Delta E_{0} \Delta E_{1}$. Thus, in the irrational case,

$$
\left\langle g_{\text {irrat }}(E)\right\rangle \propto E^{\frac{n}{2}-2} \text {. }
$$

Figure 1 shows the dependence of $\langle g(E)\rangle$, for a few examples of flat tori in $n=4$ dimensions. The linear dependence of $\langle g(E)\rangle$ at sufficiently large $E$ for rational $Q$ and its independence of energy in the irrational case are clearly illustrated.

(d) For the integer $Q, n \geqslant 4$ and in the limit of large $E$, the representing integer vectors of $E$ are uniformly distributed on the ellipsoid: given a well-behaved function $f(\mathbf{x})$ on the unit ellipsoid $\mathcal{E}=\left\{\mathbf{x} \in \mathbb{R}^{n}: \mathbf{x} \cdot Q \mathbf{x}=1\right\}$,

$$
\lim _{E \rightarrow \infty} \frac{1}{g(E)} \sum_{\mathbf{q} \cdot Q \mathbf{q}=E} f\left(\frac{\mathbf{q}}{\sqrt{E}}\right)-\int_{\mathcal{E}} f(\mathbf{x}) \mathrm{d} \mathbf{x}=0 .
$$

Some insight into the above result can be obtained from the following considerations: the average of $f\left(\frac{\mathbf{q}}{\sqrt{E}}\right)$ over the vectors which represent $E$ is given by

$$
\begin{aligned}
\frac{1}{g_{Q}(E)} \sum_{\mathbf{q} \cdot Q \mathbf{q}=E} f\left(\frac{\mathbf{q}}{\sqrt{E}}\right) & =\frac{1}{g_{Q}(E)} \int_{-\pi}^{\pi} \frac{\mathrm{d} x}{2 \pi} \mathrm{e}^{\mathrm{i} x E} \sum_{\mathbf{q} \in \mathbb{Z}^{n}} \mathrm{e}^{-\mathrm{i} x \mathbf{q} \cdot Q \mathbf{q}} f\left(\frac{\mathbf{q}}{\sqrt{E}}\right) \\
& =\frac{1}{g_{Q}(E)} \sum_{\mathbf{m} \in \mathbb{Z}^{n}} \int_{\mathbf{q} \in \mathbb{R}^{n}} \mathrm{~d}^{n} q \mathrm{e}^{2 \pi \mathrm{i} \mathbf{m} \cdot \mathbf{q}} \int_{-\pi}^{\pi} \frac{\mathrm{d} x}{2 \pi} \mathrm{e}^{\mathrm{i} x(E-\mathbf{q} \cdot Q \mathbf{q})} f\left(\frac{\mathbf{q}}{\sqrt{E}}\right) \\
& =I_{0}(E ; Q)+\sum_{\mathbf{m} \in \mathbb{Z}^{n} / 0} I_{\mathbf{m}}(E ; Q) .
\end{aligned}
$$




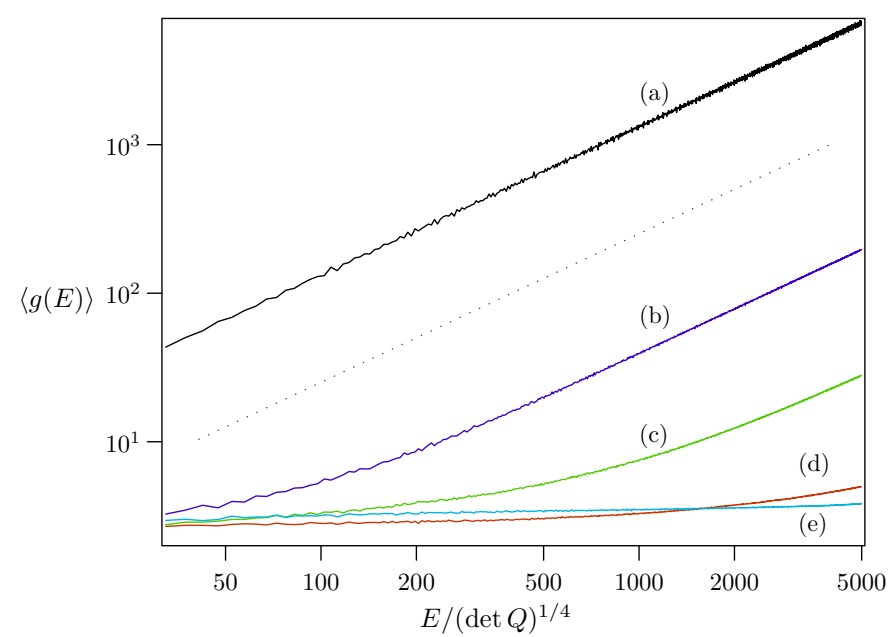

Figure 1. Double logarithmic plot of the spectral averaged degeneracy (4). The five data sets correspond to tori in four dimensions (11): (a) $\beta=2$, (b) $\beta=3 / 2$, (c) $\beta=5 / 3$, (d) $\beta=8 / 5$ and (e) $\beta=(\sqrt{5}+1) / 2$. (a) - (d) correspond to integer tori and (e) to an irrational. The parameter $\beta$ is explained in the text at the end of section 2.2. The dotted line is a linear function of $E$ included for comparison.

In particular,

$$
I_{0}(E ; Q)=\frac{2(\operatorname{det} Q)^{\frac{1}{2}}}{\Omega} \int_{\mathbb{R}^{n}} \mathrm{~d}^{n} x \delta(1-\mathbf{x} \cdot Q \mathbf{x}) f(\mathbf{x}) .
$$

Here, $\Omega$ is the volume of $\mathbb{S}^{n-1}$. The average of $f$ over the representing vectors depends on $E$ in a very complicated way. $I_{0}(E ; Q)$ is the only non-oscillatory contribution, and it provides the constant to which the average over the representations converges in the limit of large $E(7)$. The fluctuations are expressed by the sum over the $I_{\mathbf{m}}(E ; Q)$ with non-zero $\mathbf{m}$. The magnitude of these fluctuations depends on the dimension $n$, and various estimates can be found in the literature. In the present study, for the most interesting case of $n=4$, the fluctuations are bounded by $\mathcal{O}\left(E^{-\frac{1}{2}}\right)$ [21]. Being a sum of oscillatory functions, the magnitude of the fluctuations can be reduced by spectral averaging.

In the following sections, we shall consider functions $f(\mathbf{q})$ which are homogeneous of order $\mu$ and average over the representations written as

$$
F(E)=\frac{1}{g(E)} \sum_{\mathbf{q} \cdot Q \mathbf{q}=E} f(\mathbf{q})
$$

The non-oscillatory part of $F(E)$ (to be denoted by $\overline{F(E)}$ ) increases as $E^{\frac{\mu}{2}}$ and the magnitude of the fluctuations about $\overline{F(E)}$ is $\mathcal{O}\left(E^{\frac{\mu}{2}-\frac{1}{2}}\right)$ (for $n=4$ ). The systematic study of the way how the spectral average $\langle F(E)\rangle$ approaches $\overline{F(E)}$ is complicated by the fact that the series (8) is not absolutely convergent. Therefore, not much is known about this subject, and the detailed study of this issue is beyond the scope of the present work. However, in the numerical simulations we checked that the parameter $\Delta E$ can be chosen such that the spectral average approaches the smooth part to the desired accuracy. This is illustrated in figure 1, where the averaged degeneracy approaches the expected smooth power law. 


\subsection{Isospectral flat tori in four dimensions}

The four-parameter family of nonisometric yet isospectral flat tori which will be studied in most of the examples to be discussed here was discovered by Conway and Sloane [18]. It includes, as a particular case, the previously known example of Schiemann [12]. The spectra are given explicitly by the pairs of positive definite matrices, which depend on the four positive parameters $a, b, c, d$ :

$$
\begin{gathered}
Q^{+}=\frac{1}{12}\left(\begin{array}{cccc}
9 a+b+c+d & 3 a-3 b-c+d & 3 a+b-3 c-d & 3 a-b+c-3 d \\
3 a-3 b-c+d & a+9 b+c+d & a-3 b+3 c-d & a+3 b-c-3 d \\
3 a+b-3 c-d & a-3 b+3 c-d & a+b+9 c+d & a-b-3 c+3 d \\
3 a-b+c-3 d & a+3 b-c-3 d & a-b-3 c+3 d & a+b+c+9 d
\end{array}\right) \\
Q^{-}=\frac{1}{12}\left(\begin{array}{cccc}
9 a+b+c+d & -3 a+3 b-c+d & -3 a+b+3 c-d & -3 a-b+c+3 d \\
-3 a+3 b-c+d & a+9 b+c+d & a+3 b-3 c-d & a-3 b-c+3 d \\
a+b+3 c-d & a+3 b-3 c-d & a+b+9 c+d & a-b+3 c-3 d \\
-3 a-b+c+3 d & a-3 b-c+3 d & a-b+3 c-3 d & a+b+c+9 d
\end{array}\right) .
\end{gathered}
$$

Several properties of these matrices can be derived by straightforward computations:

(1) The spectra of $Q^{+}$and $Q^{-}$are identical, and consist of the values $a, b, c, d$. The unitary matrices which bring $Q^{ \pm}$to diagonal form are independent of the parameters. Explicitly,

$$
\begin{aligned}
& D \equiv \operatorname{diag}\{a, b, c, d\}=T^{ \pm} Q^{ \pm}\left(T^{ \pm}\right)^{\top} \\
& T^{ \pm}=\frac{1}{\sqrt{12}}\left[\left(\begin{array}{cccc}
0 & 1 & 1 & 1 \\
-1 & 0 & -1 & 1 \\
1 & -1 & 0 & 1 \\
1 & 1 & -1 & 0
\end{array}\right) \pm 3\left(\begin{array}{cccc}
1 & 0 & 0 & 0 \\
0 & 1 & 0 & 0 \\
0 & 0 & -1 & 0 \\
0 & 0 & 0 & -1
\end{array}\right)\right] .
\end{aligned}
$$

(2) $Q^{+}$and $Q^{-}$commute only when at least three of the parameters $a, b, c, d$ are equal. From now on when we shall refer to the four-parameter family of isospectral tori, we shall exclude this set which represents ellipsoids with cylindrical or spherical symmetry.

(3) The unitary matrix $U$ which transforms $Q^{+}$to $Q^{-}, Q^{-}=U^{\top} Q^{+} U$ is independent of the parameters $a, b, c, d$,

$$
U=\left(T^{+}\right)^{\top} T^{-}=\frac{1}{2}\left(\begin{array}{cccc}
-1 & 1 & 1 & 1 \\
-1 & -1 & -1 & 1 \\
-1 & 1 & -1 & -1 \\
-1 & -1 & 1 & -1
\end{array}\right), \quad \operatorname{det} U=1
$$

(4) The lattice vectors which define the unit cells of the tori cannot be partitioned into mutually orthogonal subsets.

Consider an integer vector $\mathbf{q}$ with $\mathbf{q} \cdot Q^{-} \mathbf{q}=E$. $U \mathbf{q}$ is on the ellipsoid generated by $Q^{+}$, but it is an integer only if $\sum_{i=1}^{4}\left|q_{i}\right|$ is even. In this case also $\sum_{i=1}^{4}\left|(U \mathbf{q})_{i}\right|$ is even. Hence, integer vectors with even sums map to each other under $U$. The integer vectors with odd sums do not have this property.

Conway and Sloane's paper offers another family of isospectral tori in four dimensions. This is a two-parameter family which we shall not discuss in detail, although the numerical results obtained for this case support the conclusions derived from the study of the fourparameter family.

To end this section we would like to describe the numerical simulations which accompany the subsequent discussions. We have calculated the first 120 million eigenvalues for five pairs of 
isospectral tori (together with the corresponding nodal sequences defined in the next section). Four of the pairs have been chosen rational, one irrational. The four parameters that define a pair (see equation (11)) are all of the form $(a, b, c, d)=\left(\alpha, \alpha / \beta^{2}, \alpha / \beta^{4}, \alpha / \beta^{6}\right)$. Here $\alpha$ just rescales the spectrum; for the rational tori it is taken such that the tori are actually integer. For the irrational pair it has been set to $\alpha=1$. Note that we present all results as a function of $E /(\operatorname{det} Q)^{1 / 4}$ which is invariant under rescalings of the spectrum. For the other parameter we have chosen the five values $\beta_{i}=2,3 / 2,5 / 3,8 / 5,(\sqrt{5}+1) / 2$. The last value defines the irrational pair $\left(\beta_{5}=(\sqrt{5}+1) / 2\right.$ is the golden ratio), the other parameters are rational approximants to the golden ratio along the Fibonacci sequence. Integer tori are obtained by setting $\alpha_{i}=2^{8}, 2^{2} \times 3^{7}, 3 \times 5^{6}, 2^{20}$ (which results in $\operatorname{det} Q\left(\alpha_{i}, \beta_{i}\right)=$ $2^{20}, 3^{16} \times 2^{20}, 3^{16} \times 5^{12}, 2^{44} \times 5^{12}$ ).

\section{Nodal domains and isospectrality}

In this section we shall define the nodal sequences of the flat tori under consideration, and will show how they can resolve isospectrality.

To define the nodal domains, we consider the real counterparts of (1),

$\Psi_{\mathbf{q}}^{(+)}=\cos \left(2 \pi \sum_{r=1}^{n} q_{r}\left(\hat{\mathbf{g}}^{(r)} \cdot \mathbf{x}\right)\right), \quad \Psi_{\mathbf{q}}^{(-)}=\sin \left(2 \pi \sum_{r=1}^{n} q_{r}\left(\hat{\mathbf{g}}^{(r)} \cdot \mathbf{x}\right)\right)$

and to avoid double counting, we must exclude $-\mathbf{q}$ if $\mathbf{q}$ is included.

A convenient representation can be obtained by the transformation

$$
\mathbf{y}=G \mathbf{x}, \quad \mathbf{x} \in T, \quad \mathbf{y} \in T_{y}=\mathbb{R}^{n} / \mathbb{Z}^{n}
$$

so that

$$
\Delta_{y}=-\sum_{r, s=1}^{n} Q_{r, s} \frac{\partial^{2}}{\partial y_{r} \partial y_{s}}
$$

and

$$
\Psi_{\mathbf{q}}^{(+)}(\mathbf{y})=\cos (2 \pi(\mathbf{q} \cdot \mathbf{y})), \quad \Psi_{\mathbf{q}}^{(-)}(\mathbf{y})=\sin (2 \pi(\mathbf{q} \cdot \mathbf{y})) .
$$

The flat tori which we consider here cannot be reduced to mutually orthogonal subspaces (see comment (iv) in the previous section). Therefore, the real wave functions (17) cannot be expressed in a product form.

We define the number of nodal domains by lifting the wave functions to $\mathbb{R}^{n}$ and counting their nodal domains in the unit cell. The nodal domains form parallel strips bounded by $(n-1)$-dimensional nodal hyper-plane and the unit cell's boundaries (figure 2$)$. The number of nodal domains is

$\hat{v}[\mathbf{q}]=2 \sum_{r=1}^{n}\left|q_{r}\right| \quad$ for $\Psi_{\mathbf{q}}^{(-)} \quad$ and $\quad \hat{v}[\mathbf{q}]=1+2 \sum_{r=1}^{n}\left|q_{r}\right| \quad$ for $\Psi_{\mathbf{q}}^{(+)}$.

This result can be proved by induction, and we start by assuming that all the $q_{i}$ are positive. For the $\Psi_{\mathbf{q}}^{(-)}(18)$ is trivially true for $n=1$. In $2 \mathrm{D}$, the nodal manifold are lines which are perpendicular to the direction $\left(q_{1}, q_{2}\right)$. There are $2 q_{1}$ such lines which intersect the unit interval on the $y_{1}$ axis at the points which are the nodal manifolds of the 1D problem. There are additional $2 q_{2}-1$ lines which intersect the interval $1<y_{2}<1$ on the line $y_{1}=1$. In total there are $2\left(q_{1}+q_{2}\right)-1$ nodal lines in the unit interval and therefore $2\left(q_{1}+q_{2}\right)$ nodal domains. The same argument can now be repeated for any $n$. The case when some $q_{i}$ are negative can be taken care of by a proper reflection. The additional 1 for $\Psi_{\mathbf{q}}^{(+)}$ 


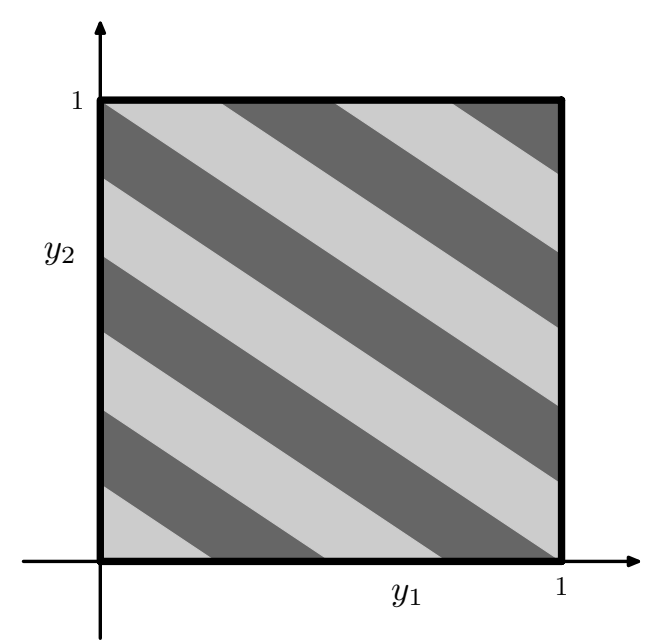

Figure 2. Nodal domains in two dimensions for $\mathbf{q}=(2,3)$. The domains are bounded inside the rectangular unit cell as explained in the text.

follows from the fact that the wave function does not vanish at the origin. In what follows, we shall count the number of nodal domains for both $\Psi_{\mathbf{q}}^{(+)}(\mathbf{y})$ and $\Psi_{\mathbf{q}}^{(-)}(\mathbf{y})$.

The strip structure discussed above is very different from the morphology of nodal domains in separable systems, which are formed from intersections of locally perpendicular hyper-planes.

The special feature of the flat tori to be discussed here is that their spectra are degenerate, and the degeneracies are maximal when the tori are integer. Any linear combination of the eigenfunctions in the degenerate space is itself an eigenfunction, and its nodal structure may depend on the particular combination. To be definite, we must choose a unique representation of the wave functions, for which the nodal count can be defined in an unambiguous way. For this purpose we require that the eigenfunctions are presented in the form of a single term and the nodal domains are parallel strips in the unit cell. This definition singles out a well-defined basis for each degeneracy space, and the number of nodal domains is given by (18).

\subsection{Counting nodal domains}

In a previous work [22], we have shown that counting nodal domains can reveal important information concerning the classical dynamics of the system under study. In particular, arranging the spectrum by increasing values of the eigenvalues, we studied the distribution of the normalized nodal counts $\xi_{n}=\frac{\hat{v}_{n}}{n}$, where $\hat{v}_{n}$ is the number of nodal domains of the $n$th wave function. Courant's theorem guaranties that $\xi_{n} \leqslant 1$. The $\xi$ distributions have characteristic and universal form if the problem is separable, which is distinct from the distribution which prevails when the classical dynamics is chaotic.

As long as the degeneracy in the spectrum is accidental, one can ignore the ambiguity induced in the ordering of the spectrum by the degeneracy. In the present case, the degeneracy increases as a power of $E$, and therefore a different quantity, which is independent of the ordering of the wave functions within a degeneracy subspace, is called for.

A natural modification of the normalized nodal counts for a highly degenerate spectrum is obtained by considering the quantity 
$v_{Q}(E)=\frac{1}{g_{Q}(E)} \sum_{\mathbf{q} \in \mathbb{Z}^{n}: E=\mathbf{q}: Q \mathbf{q}} \hat{v}[\mathbf{q}]=\frac{1}{2}+\frac{1}{g_{Q}(E)} \sum_{\mathbf{q} \in \mathbb{Z}^{n}: E=\mathbf{q} \cdot Q \mathbf{q}}\left[2 \sum_{i=1}^{n}\left|q_{i}\right|\right]$.

We shall refer to $v_{Q}(E)$ as the nodal count associated with the degenerate eigenvalue $E$. It coincides with the standard definition for nondegenerate cases. The nodal count is the tool by which we propose to resolve isospectrality. It is defined as an average over the representing vectors on the surface of the ellipsoid $E=\mathbf{q} \cdot Q \mathbf{q}$. Following the discussion in subsection (2.1), the non-oscillatory part of $v_{Q}(E)$ assumes the form

$$
\overline{v_{Q}(E)} \propto E^{\frac{1}{2}} \int_{\mathbb{R}^{4}} \mathrm{~d} \mathbf{s} \delta(1-\mathbf{s} \cdot Q \mathbf{s}) \hat{v}[\mathbf{s}]=E^{\frac{1}{2}} \int_{\mathbb{S}^{3}} \frac{\mathrm{d} \boldsymbol{\omega} \hat{v}[\boldsymbol{\omega}]}{(\boldsymbol{\omega} \cdot Q \cdot \boldsymbol{\omega})^{5 / 2}},
$$

where the factor $E^{\frac{1}{2}}$ is due to the linear dependence of $\hat{v}[\mathbf{q}]$ on $\mathbf{q}$. Because of this factor, the fluctuations in $v_{Q}(E)$ as defined above do not vanish in the large $E$ limit. However, the spectral average reduces the fluctuations and $\left\langle v_{Q}(E)\right\rangle$ approaches $\overline{v_{Q}(E)}$. This was confirmed in the numerical simulations.

\subsection{Nodal counts and isospectrality}

In this section we shall summarize the evidence we have in support of the conjecture that the sequences of nodal counts of tori which are isospectral but not isometric are different. Denoting by $Q^{+}$and $Q^{-}$the corresponding pair of Gramm matrices, and by $E$ a spectral point, we study the difference

$$
\delta v(E)=v_{Q^{+}}(E)-v_{Q^{-}}(E) .
$$

Rather than examining (21) for individual eigenvalues, we shall consider its average over spectral intervals and the fluctuations about the mean.

In the appendix we prove that

$$
\overline{\delta v(E)}=\overline{v_{Q^{+}}(E)}-\overline{v_{Q^{-}}(E)}=0 .
$$

Using the arguments given above, and detailed numerical checks, we conclude that $\langle\delta v(E)\rangle \rightarrow 0$ for large $E$.

The fluctuations in the difference of the nodal sequences are best demonstrated by studying the variance

$\left\langle(\delta v(E))^{2}\right\rangle=\frac{1}{M} \sum_{\left|E-E_{m}\right|<\Delta / 2}\left(v_{Q^{+}}\left(E_{m}\right)-v_{Q^{-}}\left(E_{m}\right)\right)^{2}, \quad E_{m}$ in the spectrum.

Here $\Delta \ll E$ and $M=\sharp\left\{E_{m}:\left|E-E_{m}\right|<\Delta / 2\right\}$. The variance $\left\langle(\delta v(E))^{2}\right\rangle$ was computed for the examples of nonisometric yet isospectral tori discussed in conjunction with figure 1 . The results are shown in figure 3. The variance does not vanish, and its dependence on $E$ clearly distinguishes between the rational and irrational pairs. The variance for the irrational $Q^{ \pm}(\beta=(\sqrt{5}+1) / 2)$ increases linearly with $E$, while the variance for the most rational case $(\beta=2)$ fluctuates about a constant value. The data for the intermediate sets of parameters increase linearly at low $E$, and approach constant values after $E$ is large enough to 'distinguish' that the coefficients are rational. The same behaviour was also observed for the two-parameter family of isospectral tori in four dimensions mentioned in [18].

We are unable to prove that the variance (23) differs from zero, and that its $E$ dependence follows the behaviour observed in the numerical simulations. However, we can provide heuristic arguments which explain the systematic trends observed in the data. The underlying assumptions are that 


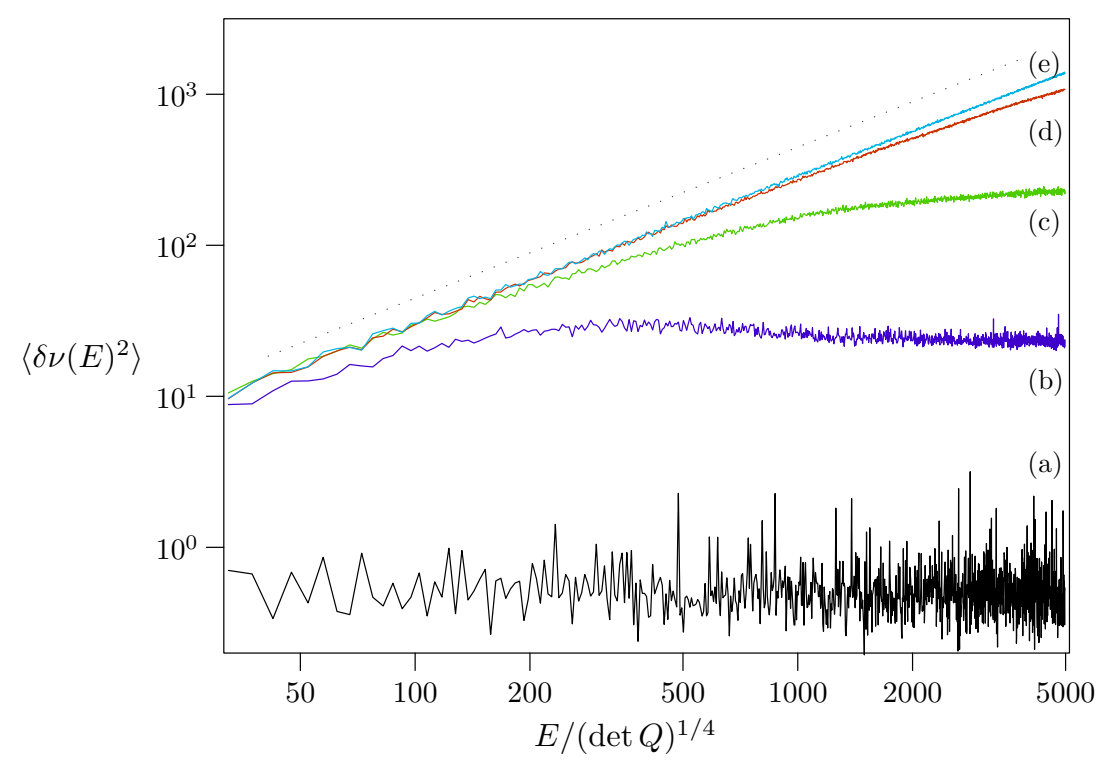

Figure 3. Double logarithmic plot of the variance $\left\langle\delta v(E)^{2}\right\rangle(23)$. The parameters are the same as in figure 1 . The dashed line is a linear function of $E$ included for comparison.

(I) the number of nodal domains for individual representing vectors $\hat{v}[\mathbf{q}]$ (18) fluctuate independently about their mean (20);

(II) the fluctuations in $\delta v_{Q^{+}}(E)$ and $\delta v_{Q^{-}}(E)$ are also independent. (This is supported by the observation made in point (iii) of the preceding section that only a fraction of the representation vectors of the two Gram matrices can be transformed to each other by a single rotation.)

Consider first the functions $v_{Q}(E)$ separately for $Q=Q^{+}$and $Q=Q^{-}$. The first assumption together with the central limit theorem implies that the variance of the fluctuations about their mean $\left\langle\left(v_{Q}(E)-\left\langle v_{Q}(E)\right\rangle\right)^{2}\right\rangle$ is inversely proportional to the number of terms in the sum which is the mean degeneracy $\left\langle g_{Q}(E)\right\rangle$. The magnitude of the fluctuation is also proportional to $E^{\frac{1}{2}}$ since $v(E)$ scales this way. Thus, we expect

$$
\left\langle\left(v_{Q}(E)-\left\langle v_{Q}(E)\right\rangle\right)^{2}\right\rangle \propto \frac{E}{\left\langle g_{Q}(E)\right\rangle} \quad \text { for } \quad Q=Q^{+}, Q^{-} .
$$

We expect this behaviour to hold for both rational and irrational tori. For rational tori, this relation is consistent with number of theoretical estimates, which were mentioned in section (2), point (d). Using the second assumption, we conclude that

$$
\left\langle\left(\delta v_{Q}(E)\right)^{2}\right\rangle \propto \frac{E}{\left\langle g_{Q}(E)\right\rangle} .
$$

Thus, for rational $Q^{ \pm}$where $\left\langle g_{Q}(E)\right\rangle \propto E$, the variance is independent of $E$, whereas for irrational $Q^{ \pm}$the variance is expected to vary linearly with $E$. This behaviour is indeed observed in the numerical simulations.

Another numerical test which supports the validity of the above analysis, consisted in computing the fluctuations of the integrated nodal counts

$$
N_{Q}(E)=\frac{1}{\mathcal{N}(E)}\left(\sum_{\mathbf{q} \in \mathbb{Z}^{n}: \mathbf{q} \cdot Q \mathbf{q} \leqslant E} \hat{v}[\mathbf{q}]\right),
$$




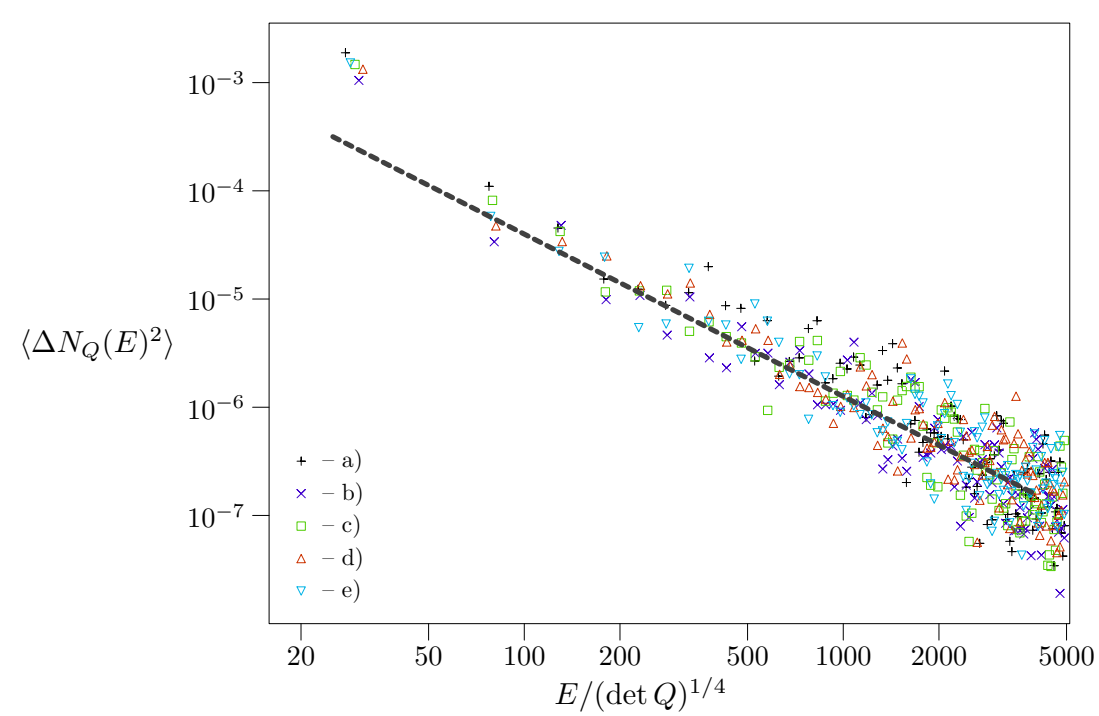

Figure 4. Double logarithmic plot of the squared nodal count difference $\left\langle\Delta N(E)^{2}\right\rangle$ (smoothed by taking an average over a small spectral interval $\Delta E$ ). The parameters are the same as for figure 1 . The dotted line shows the theoretical prediction $E^{-3 / 2}$.

where $\mathcal{N}(E) \sim E^{2}$ is the spectral counting function $\mathcal{N}(E)=\sum_{e \leqslant E} g_{Q}(e)$. The difference between integrated nodal counts for the isospectral pair can be written as

$$
\Delta N(E)=N_{Q^{+}}(E)-N_{Q^{-}}(E)=\frac{1}{\mathcal{N}(E)}\left(\sum_{\mathbf{q} \in V_{+}} \hat{v}[\mathbf{q}]-\sum_{\mathbf{q} \in V_{-}} \hat{v}[\mathbf{q}]\right),
$$

where $V_{ \pm}:=\left\{\mathbf{q} \in \mathbb{Z}: \mathbf{q} \cdot Q_{ \pm} \mathbf{q} \leqslant E\right.$ and $\left.\mathbf{q} \cdot Q_{\mp} \mathbf{q}>E\right\}$. Here, we made use of the exact cancellations of the contributions of $\mathbf{q} \in \mathbb{Z}$ in the intersection of the two ellipsoids $\mathbf{q} \cdot Q_{+} \mathbf{q} \leqslant E$ and $\mathbf{q} \cdot Q_{-} \mathbf{q} \leqslant E$. There are further exact cancellations since $\hat{v}[\mathbf{q}]$ is invariant under reflections $q_{i} \rightarrow-q_{i}$ and due to the symmetries between the two ellipsoids. Assuming that uncorrelated contributions to $\Delta N(E)$ stem from a thin layer at the surface of the two ellipsoids while bulk contributions cancel we have a sum over $s \sim|\mathbf{q}|^{3}=E^{3 / 2}$ independent contributions of order $\hat{v}[\mathbf{q}] \sim E^{1 / 2}$ that vanishes in the mean. For the variance this assumption leads to

$$
\left\langle N_{Q}(E)^{2}\right\rangle \sim \frac{s \hat{v}[q]^{2}}{\mathcal{N}(E)^{2}} \sim E^{-3 / 2}
$$

in accordance with our numerical analysis (see figure 4). Note that this result is valid for both rational and irrational tori.

\section{Summary and conclusions}

The heuristic arguments as well as the numerical evidence collected above, support our conjecture that isospectral flat tori can be distinguished by studying the fluctuations of their nodal counts. A formal proof is still lacking. At the same time, the work indicates a hitherto unnoticed link between isospectrality and nodal counts. We hope that the present work will induce more research effort in this direction. 
The nodal count studied here is a particular function of the representation vectors whose fluctuations distinguish between the isospectral tori. Are there any other functions which are sensitive to these differences? We investigated this question to some extent, and were not able to find a simple criterion which the function should satisfy in order to resolve the spectral ambiguity. The nodal count was not chosen arbitrarily, and it is rewarding that it does have the required property.

As was mentioned above, there exist several other known families of flat tori beyond the ones discussed here at length. The way of reasoning proposed above should apply to these cases as well. Exploratory work in this direction shows that the nodal sequences of isospectral pairs are different for the cases that were tried, but a systematic study gets prohibitively time consuming as the dimension increases.

The conjecture that nodal counts resolve isospectrality is now being tested for a different class of operators - the Schrödinger operators on quantum graphs [23]. Preliminary results support the validity of the conjecture also for this class of operators which are quite different from the flat tori discussed here.

\section{Acknowledgments}

This work was supported by the Minerva Center for Nonlinear Physics, the Einstein (Minerva) Center at the Weizmann Institute and by ISF and GIF research grants. NS acknowledges a post doctoral fellowship from the European Network on Mathematical aspects of Quantum Chaos which supported his stay at the Weizmann Institute. US acknowledges support from the EPSRC grant GR/T06872/01. We would like to thank Professor Zeev Rudnick for several crucial comments and discussions, and to Professor Jochen Brüning for discussions during the early stages of the project.

\section{Appendix. The mean nodal counts of isospectral tori}

To demonstrate the equality of the smooth parts consider the simpler quantity

$$
\tau_{Q}=\int_{\mathbb{S}^{3}} \mathrm{~d} \boldsymbol{\omega} \sum_{i}\left|\omega_{i}\right| \cdot \frac{1}{(\boldsymbol{\omega} \cdot Q \cdot \boldsymbol{\omega})^{\eta}}
$$

with $\eta$ being some power e.g. 5/2. The goal is to show $\tau_{Q^{+}}=\tau_{Q^{-}}$. From the diagonalization (12):

$$
Q^{ \pm}=\left(T^{ \pm}\right)^{\top} D T^{ \pm}
$$

make a change of variables

$$
\mathbf{o}=T^{ \pm} \cdot \omega .
$$

This preserves the measure as $T^{ \pm}$are orthogonal and the denominators are seen to agree as $D$ is the same for the two lattices. The numerator becomes

$$
\sum_{i}\left|\omega_{i}\right|=\sum_{i, j}\left|\left(T^{ \pm}\right)_{i j}^{\top} o_{j}\right|=\sum_{i, j}\left|T_{j i}^{ \pm} o_{j}\right| .
$$

By explicit calculation we get for the '+' lattice:

$$
\begin{aligned}
\sqrt{12} \sum_{i}\left|\omega_{i}\right|= & \left|o_{1}+o_{2}+o_{3}-3 o_{4}\right|+\left|o_{1}-o_{2}-3 o_{3}-o_{4}\right| \\
& +\left|o_{1}+3 o_{2}-o_{3}+o_{4}\right|+\left|3 o_{1}-o_{2}+o_{3}+o_{4}\right|
\end{aligned}
$$


and similarly for the '-' lattice,

$$
\begin{aligned}
\sqrt{12} \sum_{i}\left|\omega_{i}\right|= & \left|o_{1}-o_{2}+3 o_{3}-o_{4}\right|+\left|o_{1}-3 o_{2}-o_{3}+o_{4}\right| \\
& +\left|-3 o_{1}-o_{2}+o_{3}+o_{4}\right|+\left|o_{1}+o_{2}+o_{3}+3 o_{4}\right|
\end{aligned}
$$

Each absolute value for one lattice can be made to correspond to the other by changing $o_{i}$ to $-o_{i}$. For instance the first term of the ' + ' lattice matches with the fourth of the ' - ' lattice. In the integral for $\tau$ such sign changes preserve both the integration domain, the measure and the denominator. Therefore when splitting the integral into four, corresponding to the terms of (A.5), (A.6), each of these terms can be brought to an agreement.

\section{References}

[1] Kac M 1966 Am. Math. Mon. 73 1-23

[2] Brüning J and Heintze E 1984 Math. Ann. 26995

[3] Zelditch S 1998 J. Diff. Geom. 49207

[4] Gutkin B and Smilansky U 2001 J. Phys. A: Math. Gen. 31 6061-8

[5] Zelditch S 2005 Survey on the inverse spectral problem J. Diff. Geom. Surveys to appear

[6] Milnor J 1964 Proc. Natl Acad. Sci. USA 51542

[7] Fisher M E 1966 J. Comb. Theor. 1 105-25

[8] Gordon C, Webb D and Wolpert S 1992 Bull. Am. Math. Soc. 27 134-8

[9] Chapman J S 1995 Am. Math. Mon. 102124

[10] Sunada T 1985 Ann. Math. 121 196-86

[11] Brooks R 1999 Ann. Inst. Fourier 49 707-25

[12] Schiemann A 1990 Arch. Math. 54372

[13] Buser P, Conway J, Doyle P and Semmler K-D 1994 Int. Math. Res. Not. 9 391-400

[14] Okada Y and Shudo A 2001 J. Phys. A: Math. Gen. 34 5911-22

[15] Kneser M 1967 Math. Ann. 11831

[16] Wolpert S 1978 Trans. Am. Math. Soc. 244313

[17] Earnest C R and Nipp G 1991 Math. Rep. Acad. Sci. Can. 1333

[18] Conway J H and Sloane N J A 1992 Duke Math. J. 6693

[19] Cassels J W S 1978 Rational Quadratic Forms (London: Academic)

[20] Iwaniec H 1997 Topics in Classical Automorphic Forms (Providence, RI: American Mathematical Society)

[21] Rudnick Z 2004 private communication

[22] Blum G, Gnutzmann S and Smilansky U 2002 Phys. Rev. Lett. 88114101

[23] Shapira T and Smilansky U 2005 Quantum graphs which sound the same Nonlinear Dynamics and Fundamental Interactions (Proc. Nato Advanced Research Workshop, Tashkent 2004) ed F Khanna and D Matrasulov (Berlin: Springer) 